\title{
MOVIMIENTO NACIONAL DE POLÍTICAS PÚBLICAS DE EDUCACIÓN DOCENTE: los casos de Brasil y Uruguay
}

\author{
Regina Magna Bonifácio de Araújo \\ regina.araujo@ufop.edu.br \\ https://orcid.org/0000-0001-7289-5876 \\ Universidade Federal de Ouro Preto \\ Minas Gerais, Brasil. \\ Eduardo Rodriguez Zidan \\ cerzidan@yahoo.com.ar \\ https://orcid.org/0000-0002-6437-578X \\ Universidad ORT \\ Montevideo, Uruguay
}

Recibido: 01/11/2020 Aceptado: 01/12/2020

\begin{abstract}
Resumen
Este artículo tiene como objetivo presentar políticas públicas para la formación del profesorado de Educación Básica en Brasil y Uruguay, destacando el movimiento de cada conjunto legislativo, su institución y su alcance. Guiado por la comprensión de la complejidad existente en la realidad de cada país, el texto aborda el tema de la capacitación, los desafíos existentes y los avances logrados en cada contexto educativo.
\end{abstract}

Palabras clave: Formación del profesorado; Políticas públicas; Legislación educativa; Formación inicial; Modelos educativos.

\section{MOVIMENTO DAS POLÍTICAS NACIONAIS DE FORMAÇÃO DOCENTE:}

\section{Os casos de Brasil e Uruguai}

\section{Resumo}

O presente artigo tem como objetivo apresentar as políticas públicas de formação de professores para a Educação Básica existentes no Brasil e no Uruguay, destacando o movimento de cada conjunto legislativo, sua instituição e seus alcances. Guiados pela compreensão da complexidade existente na realidade de cada país, o texto aborda a problemática da formação, os desafios existentes e os avanços conquistados a partir de cada contexto educacional.

Palavras chave: Formação de professor; Políticas públicas; Legislação educacional; Formação inicial; Modelos educativos. 


\title{
NATIONAL TEACHER EDUCATION POLICY MOVEMENT:
}

\section{The cases of Brazil and Uruguay}

\begin{abstract}
In this paper we report a synthesis of a reconstruction of the derivative holistic meaning carry out using some theoretical notions of the onto-semiotic approach. We characterize the intended high school curricular meaning about the derivative, based on the mathematical practices proposed in both the core curriculum and in the textbooks. The comparison between the global and curricular meanings allows assessing the "epistemic suitability of curricular meaning", intended for the high school curriculum. The methodology of the didactical analysis, applied to the case of the derivative in the Mexican curriculum and textbook can be extended to other contents and contexts. The information provided may be useful for high school mathematics teacher because we reveal some biases in the meanings of the derivative privileged by the curriculum that could be avoided to improve the teaching of the derivative.

Keywords: Derivative. Holistic Meaning. Mathematics Curriculum. Teacher Knowledge. Ontosemiotic Approach.
\end{abstract}

\section{Introducción}

En los últimos años, la producción académica y los debates sobre la formación del profesorado, tanto iniciales como continuos, han aumentado significativamente, lo que se puede ver por la considerable producción académica en el área, por disertaciones y tesis, y eventos académicos, nacionales e internacionales. La centralidad de estos estudios se basa, en general, en aspectos formales de la capacitación, temas que ya han sido ampliamente debatidos, pero que se centran poco en las políticas de capacitación y en la trayectoria que estas políticas han estado siguiendo en el escenario educativo.

Este artículo busca despertar interés en cuestionar algunas ideas en el campo de las políticas públicas de educación docente, así como informar lo que está sucediendo en Brasil y Uruguay, países vecinos y parceros en diversas iniciativas educativas. Con este fin, los autores realizarán un mapeo rápido de las políticas públicas para la formación de docentes para la Educación Básica, destacando sus orientaciones y tendencias, reflexionando sobre las realidades de ambos países y considerando la complejidad de cada locus formativo. 


\section{Una mirada a las políticas nacionales de formación docente en Brasil}

La capacitación de docentes en Brasil se ha convertido en un tema central en las discusiones sobre políticas educativas y uno de los principales desafíos que debe enfrentar la administración pública. Presentada como un campo prominente en políticas, en pautas, en la Conferencia Nacional de Educación (CONAE), en el Plan Nacional de Educación (PNE), la educación docente entra hoy trayendo algunas reflexiones que nos mueven del lugar común en la discusión sobre un entrenamiento idealizado o una condición de trabajo docente que interroga e incluso condiciona este entrenamiento (ARROYO, 2015). Sin embargo, sin embarcarnos en esta área, presentaremos en esta sección cómo estas políticas públicas se destacaron después de la Constitución de 1988 y el papel del Legislativo en la constitución de leyes y acciones para su implementación.

También es importante resaltar que "este campo se ha constituido en un contexto de tensiones y contradicciones que se establecen en un escenario con antecedentes neoliberales y se han caracterizado por la creciente intervención del poder estatal constituido" (OLIVEIRA; LEIRO, 2017, p. 01). Tensiones y contradicciones que ignoran la relación inseparable entre el trabajo docente y la formación del profesorado, y que no tienen en cuenta la diversidad y vulnerabilidad en la que viven los niños y jóvenes atendidos por estos educadores.

Desde 1990, la carrera docente ha ocupado un espacio significativo en las esferas política y académica en todo Brasil. Destacamos, inicialmente, la Ley de Directrices y Bases para la Educación Nacional - LDB n ${ }^{\text {o }}$ 9.394/96, en los artículos 61 y 62 cuando se refiere específicamente a la formación docente. El artículo 61 presenta los fundamentos de esta capacitación, destacando la asociación necesaria entre la teoría y la práctica y la importancia de reconocer las experiencias previas de los educadores. El artículo 62 establece que la formación del profesorado, para trabajar en Educación Básica, debe tener lugar en un nivel superior, en cursos de pregrado, y admite la formación en un nivel medio, en el modo Normal, para trabajar en Educación Infantil y en los primeros grados de Enseñanza. Fundamental También destacamos el artículo 63 que inicialmente reguló la capacitación que pueden ofrecer los Institutos de Educación Superior (ISE): curso Superior Normal, cursos para aquellos que ya tienen un título universitario y desean trabajar en Educación Básica (EB) y programas de educación continua. En abril de 1999, la Resolución CNE / CEB no 2 de 1999, instituyó las 
"Directrices Curriculares Nacionales para la Capacitación de Maestros en Educación Infantil y en los primeros años de la Escuela Primaria, en el Nivel Medio, en el modo Normal", cursos cada día menos ofrecidos en territorio nacional.

Desde su inicio, el siglo XXI ha estado marcado por políticas públicas que buscan reformular los procesos de capacitación docente en el país. En 2001, se aprobó la Ley n ${ }^{\circ} 10.172$, de 2001, que aprobó el Plan Nacional de Educación, con pautas y objetivos para los próximos diez años. Entre estas pautas, las pautas específicas para la capacitación de docentes indicaron la implementación de políticas públicas como condición para el desarrollo científico y tecnológico del país y establecieron un plazo de un año para la construcción de pautas y parámetros curriculares para cursos de educación superior para docentes. Luego, el Consejo Nacional de Educación publicó estas pautas a través de las Resoluciones CNE/CP n 01 y 02, en 2002. En el ámbito de la regulación de las pautas de los cursos que forman los docentes de Educación Básica, en 2006 el Consejo también publicó la Resolución CNE/CP n n 01/2006, que definió las Directrices Curriculares Nacionales para el Curso de Pregrado en Pedagogía, licenciatura, que regula el desempeño de los docentes para la Educación Infantil y los primeros años de la Escuela Primaria.

Para Brzezinski (2007), esta Resolución indica los elementos normativos que son consensuados en el diálogo sobre la enseñanza como base de la identidad del pedagogo; base nacional común como núcleo esencial de la formación; formación del pedagogo más completo; formación para gestión educativa e investigación; calificaciones extintas; posibilidad de superar la organización curricular por materia y también define la experiencia docente como un requisito previo para la práctica profesional en otras funciones. Esta posición no fue compartida por otros teóricos y académicos en el campo.

La creación de la Universidade Aberta do Brasil - UAB, mediante el Decreto n ${ }^{\circ} 5.800$, de 8 de 2006, para la provisión de Educación a Distancia (DE), con el objetivo de expandir e internalizar cursos y programas de Educación Superior en Brasil, principalmente aquellos centrados en la formación del profesorado. Ya hay algunos estudios sobre este tipo de capacitación, pero aún queda mucho por estudiar. "Se debe tener cuidado de que, bajo el discurso de la innovación, la transposición simple de los métodos tradicionales no se lleve a cabo en 
nuevos entornos de aprendizaje, que reproducen, con nuevas tecnologías, viejos paradigmas educativos" (OLIVEIRA; LEIRO, 2019, p. 7)

Desde 2000, el Estado ha asumido un papel más activo en la dirección de las políticas de capacitación docente en Brasil, promoviendo acciones con instituciones públicas y creando la Política Nacional para la Capacitación de Docentes de Educación Básica, en la perspectiva de un sistema Nacional de Educion (GATTI et al., 2011). Dos movimientos fueron decisivos: la aprobación de la Ley 11.494 de 2007, que amplió FUNDEF al Fondo para el Mantenimiento y Desarrollo de la Educación Básica y la Valorización de los Profesionales de la Educación (FUNDEB), extendiendo los fondos previstos para la capacitación para Maestros de educación infantil y secundaria. Y la Ley $n^{\circ} 11.502$ de 2007, que modifica la estructura y las competencias de Capes, determinando que este organismo debería subsidiar al Ministerio de Educación en la formulación de políticas para la capacitación de maestros de Educación Básica.

La publicación del Decreto nº 6,755, de 2009, que instituyó la Política Nacional para la Capacitación de Profesionales en la Enseñanza de la Educación Básica, así como el desempeño disciplinado de Capes en estas acciones de capacitación, buscó garantizar un cierto estándar de calidad para los cursos. formación docente que promueve la articulación entre teoría y práctica y la inseparabilidad entre enseñanza, investigación y extensión. El Decreto también anuncia el reconocimiento de la escuela como un espacio de capacitación, la importancia del maestro en el proceso educativo de la escuela, así como su necesaria valorización profesional. Este aparato legal avanza en el sentido de proponer la articulación entre la formación inicial y continua y entre los niveles de educación. Para enfrentar estos desafíos, se crearon los Foros Estatales Permanentes para Apoyar la Educación de Maestros, regulados por la Ordenanza n ${ }^{\circ}$ 883, de septiembre de 2009, que tienen la función de organizar la capacitación inicial y continua de los profesionales de Educación Básica. Capes, como parte de sus estrategias, creó en 2009 el Plan Nacional para la Formación de Profesores de Educación Básica - PARFOR, gestionado con el apoyo de las Secretarías de Estado y Municipales, incluso a través de los Foros (Ordenanza $n^{\mathbf{o}}$ 09, de 2009).

En 2009 se publicó un cambio importante en la LDB, con la Ley n 12.056, de 2009, que agregó tres párrafos al Artículo 62. El primero trataba sobre el régimen de colaboración entre las entidades federadas para la formación inicial y continua de los docentes. El segundo permite 
el uso de recursos y tecnologías de educación a distancia para la educación y capacitación continua de profesionales de la enseñanza y el tercer párrafo define que la capacitación inicial debe realizarse preferiblemente en persona, pero que puede recibir apoyo de los recursos y tecnologías de EaD. También en 2009, la Red Nacional de Formación Docente se designa ahora como la Red Nacional de Educación Continua para Profesionales de la Educación Básica, regulada por la Ordenanza MEC nº 1.129, de 2009. Formada por IES públicas y comunitarias sin fines de lucro y por los Institutos Federales de Educación, esta red tiene como objetivo articularse con los sistemas educativos y con los foros estatales.

Una nueva denominación llega en septiembre de 2011, la Red Nacional para la Educación Continua de Profesionales en la Enseñanza de la Educación Pública Básica, que fue instituida por la Ordenanza del MEC, $\mathrm{n}^{\mathrm{o}}$ 1.328. Esta disposición legal revocó la anterior $\mathrm{y}$ transfirió la coordinación del Comité de Gestión de la Red a la Política Nacional de Educación Inicial y Continua para Profesionales de la Educación Básica, con la función de formular, coordinar y evaluar las acciones y programas del Ministerio de Educación. Education, Capes and the National Education Development Fund (FNDE). Sin embargo, todos estos movimientos e intervenciones administrativas y políticas no tienen sentido sin la asignación necesaria de recursos para la promoción de la formación docente en línea con las demandas de educación con calidad social.

Con la aprobación del nuevo Plan Nacional de Educación, instituido por la Ley $\mathrm{n}^{\circ}$ 13.005, de 2014, válido por los próximos diez años, se indicó la institución del Sistema Nacional de Educación, en el artículo 13, que debería ser creado por ley específica hasta 2016, que será responsable, en colaboración, de monitorear la implementación de los objetivos y estrategias de este Plan. Los objetivos 15 y 16, que tratan específicamente de la capacitación de maestros de Educación Básica, establecen que, dentro de un año, todos los maestros tienen educación superior y que, al final de este Plan, el 50\% de los maestros tienen capacitación en nivel de posgrado En el documento Instituir un sistema educativo nacional: Agenda obligatoria para el país, publicado a fines del primer semestre de 2015, el MEC presentó un conjunto de propuestas articuladas para la redacción de la legislación que regirá el sistema.

Aún en 2015, la Resolución CNE / CP nº 02/2015, definió las Directrices Curriculares Nacionales para la formación inicial de nivel superior (cursos de pregrado, cursos de formación 
pedagógica para graduados y cursos de segundo grado) y para la formación continua de profesionales Educacion Basica. Con esta nueva disposición legal, buscamos asegurar una mayor organicidad para la capacitación de docentes en el país y una mayor claridad sobre el concepto de capacitación inicial (preferiblemente ofrecida en persona) y continuamos. El perfil del graduado que debe guiar la constitución y la reformulación de los cursos de pregrado en instituciones de capacitación también es una preocupación.

En 2019, el Ministerio de Educación (MEC) aprobó el Dictamen CNE / CP nº 22/2019, del Consejo Nacional de Educación (CNE), que actualizó las Directrices Curriculares Nacionales para la Formación Inicial de Maestros para Educación Básica e instituyó la Base Nacional Común para la formación inicial de docentes de educación básica, denominada formación BNC. Entre los principales aspectos aportados por las nuevas directrices, se encuentra la carga de trabajo mínima de 3.2 mil horas para todos los cursos de educación superior, destinados a la formación inicial de docentes para la educación básica. Otros aspectos importantes son la definición de cargas de trabajo diferenciadas para aquellos que toman un segundo grado y para aquellos que desean especializarse en gestión escolar, además de un curso de calificación pedagógica para graduados no graduados, con 760 horas básicas. El dictamen también estableció que las instituciones de capacitación deben organizar el proceso de evaluación de los estudiantes de pregrado "de manera continua y articulada con los entornos de aprendizaje" y que el INEP realice ajustes para contemplar los cambios en el Examen Nacional de Desempeño Estudiantil (Enade).

El documento también incluye un anexo a BNC-Training, que, siguiendo las líneas de BNCC, establece competencias generales y específicas, esta vez para los docentes. Hay 10 competencias generales y 12 competencias específicas, estas últimas agrupadas en tres dimensiones (conocimiento profesional, práctica y compromiso), con descripciones detalladas de las habilidades en cada una.

La legislación presentada en este breve informe muestra la centralización del ejecutivo federal en la regulación de las políticas de educación docente para la Educación Básica. Si bien los marcos legales que regulan actualmente la formación docente en Brasil no nos permiten concluir que existe una política estatal sólida, observamos acciones e intensidades específicas que buscan una cierta organicidad y coherencia en estos movimientos. Sin embargo, estas 
acciones, en general, no parten del consenso del área académica, ni consideran la investigación y todo el marco teórico construido hasta el momento y que podrían subsidiar una política más consistente con la realidad de la Educación Básica en el país.

Un dato que merece destacarse es la discontinuidad de acciones específicas del gobierno, como se ve en el momento actual en el país, con graves reveses y recortes en los recursos financieros, que son cada vez más importantes en educación. Sin recursos financieros y humanos, no existe una educación que pueda promover el desarrollo de los seres humanos y que garantice la formación del profesorado que articule el conocimiento y las prácticas producidas en el campo de la educación en la construcción de procesos de enseñanza y aprendizaje más coherentes y consistentes.

\section{Políticas docentes para la educación básica en Uruguay}

En esta sección se analizan la normativa y los principales instrumentos de políticas docentes para educación básica del sistema educativo uruguayo, a partir del año 1985 hasta la asunción del nuevo gobierno de la educación que asumió el 1 de marzo de 2020.

Luego de contextualizar las particularidades del sistema educativo nacional, se examina el contenido de las principales propuestas de legislación educativa en el periodo, destacando hitos, avances y desafíos pendientes.

\section{Marco contextual referido la particularidad del sistema educativo en Uruguay}

Uruguay presenta un diseño institucional del gobierno de la educación que lo distingue del resto de los países de la región. En contraposición a lo que acontece a nivel internacional, el Ministerio de Educación y Cultura de Uruguay (MEC) tiene un rol secundario respecto al diseño, implementación y evaluación de políticas educativas en general, y políticas docentes en particular.

Quien lleva a cabo la política educativa es un organismo con autonomía técnica y administrativa (no financiera) y que tiene independencia del poder político y del poder ejecutivo del gobierno de turno: la Administración Nacional de Educación Pública (ANEP). Este ente autónomo, creado por la Ley de Educación Nro. 15.739 (de emergencia educativa) en el año 
1990, tiene entre sus cometidos el diseño, implementación y evaluación de programas de formación docente para los niveles de educación primaria, secundaria, técnica y formación docente.

La ANEP, según los datos reportados por el Censo Nacional Docente (Censo, 2018) administra, regula y supervisa la enseñanza de 692.851 estudiantes y 52.387 docentes desde preescolar hasta la formación docente no universitaria. Los docentes de educación básica son aproximadamente 44.000, siendo 3.177 los formadores de docentes.

El ente autónomo ANEP es gobernado por un Consejo Directivo Central (CODICEN) cuyos miembros son designados con aval de la cámara de senadores del parlamento nacional. El presidente del CODICEN es el director nacional de educación pública (con un rango de competencias y legitimación similar a los ministros de educación del continente). El consejo que regula el ente autónomo ANEP funciona en un régimen colegiado, integrado por 5 miembros y que tiene representación, desde el año 2008, de un delegado por el orden docente. El gobierno del sistema educativo uruguayo ha sido caracterizado por su rasgos de estabilidad de las autoridades, la autonomía técnica del gobierno de la enseñanza respecto al poder político, la legitimidad institucional de tres actores fundamentales como la ANEP, MEC y la UDELAR y el cogobienro de la educación universitaria (Mancebo, 2007).

En síntesis, la particularidad de MEC es que tiene muy pocas competencias referidas a las políticas de formación inicial, perfeccionamiento, diseño de programas para educación básica, media y pre universitaria. Estas áreas de la política se llevan a cabo por entes descentralizados que no que dependen del MEC, tienen autonomía y se gobiernan por colectivas con representación de los docentes ${ }^{1}$.

\section{El período 1985- 1995: restauración democratica}

Las políticas docentes al inicio de este periodo buscaron recomponer el impacto

\footnotetext{
${ }^{1}$ El Art. 5 de la Ley de 1990 dictaminó lo siguiente “Créase la Administración Nacional de Educación Pública, Ente Autónomo con personería jurídica. Art. $7^{\circ}$.- Los órganos de la Administración Nacional La el Consejo Directivo Central, la Dirección Nacional de Educación Pública, los Consejos de Educación Primaria, de Educación Secundaria y de Educación Técnico - Profesional y sus respectivas Direcciones Generales. Se introducen câmbios en La Ley General de Educación del año 2008, vigente, que expresa en su Artículo 54. La Administración Nacional de Educación Pública tiene los siguientes órganos: el Consejo Directivo Central, los Consejos de Educación Inicial y Primaria, de Educación Media Básica, de Educación Media Superior y de Educación Técnico-Profesional (UTU).
} 
negativo de las consecuencias derivadas de la intervención del gobierno militar en los planes curriculares de enseñanza básica, la reducción del presupuesto educativo y las destituciones masivas de docentes.

En este escenario de recuperación de la democracia, se impulsaron múltiples estudios diagnósticos para conocer el estado de la educación luego de cerrarse el ciclo de la dictadura militar (entre los años 1973 y 1984). El proyecto de ley presupuesto, gastos e inversiones que elaboran las autoridades del CODICEN de la ANEP en el año 1985 (Ley de Presupuesto, Gastos e Inversiones Nero. 15.739), previo a su asunción, constituye la principal herramienta para la formulación, diseño de metas y posterior evaluación de las políticas educativas del periodo de 5 años que dura su mandato constitucional.

Las autoridades que asumieron en el año 1986, manifestaron su profunda preocupación por "las carencias, y deterioro material de los centros de enseñanza; de las menguadas asignaciones que percibe el personal docente; del anticuado, oneroso, irracional e insuficiente funcionamiento de los servicios administrativos: de los bajos sueldos de los funcionarios; de la inmediata necesidad de rectificar la orientación general de la enseñanza caracterizada por el enciclopedismo libresco y el estancamiento demostraron los estudios aludidos y las estadísticas oficiales" (ANEP, 1986, p.4)

El principal desafío para las políticas implementados post dictadura fue recomponer las estructura laboral y mejorar las condiciones materiales y de infraestructura del trabajo docente. Si bien los efectos de la intervención militar de la educación fueron extremadamente negativos, tanto a nivel de la persecución de docentes como en el cercenamiento de las libertades, desde hace décadas el país enfrenta un problema grave de déficit de la formación docente para educación básica (especialmente para los 7, 8vo y 9no, q en Uruguay denominamos ciclo básico). Este proceso de desprofesionalización docente ya se venia acentuando desde la década de $\operatorname{los} 60$ y en su momento fue reportado en profundidad por el informe elaborado por la Comisión de Inversiones y Desarrollo Económico del estado (CIDE,1965)

En los 90, el $75 \%$ de los profesores y las profesoras no poseía titulo docente , este porcentaje se elevaba a $88 \%$ en la educación media técnica. Además se constató una alta rotación de los docentes entre diferentes establecimientos, baja proporción de docentes efectivos 
en el ciclo básico de educación secundaria. La mitad de los docentes del ciclo básico (7,8 y 9no) de secundaria y tres de cada cuatro profesores de la educación técnica habían ingresado a la profesión docente por nombramiento directo, es decir, sin las debidas garantías y la legitimidad que ofrecen los concursos de oposición o méritos para el acceso a la profesión.

La estabilidad del cuerpo docente y el establecimiento de los derechos inherentes al ejercicio de la profesión docente fue una de las preocupaciones principales de las políticas públicas de la educación, tal lo expresado en diferentes documentos y reglamentos de la época:

El marco de las políticas públicas en educación en este quinquenio priorizó el diseño de la nueva estructura institucional del sistema educativo y la delimitación de las responsabilidades a nivel del gobierno educativo. Se introducen cambios en el currículo de educación básica (plan curricular 1986 que estableció la creación de un Ciclo Básico Único de 3 años) la extensión del Tiempo Pedagógico (de 27 a 32 horas), la incorporación de un curriculum por áreas en sustitución del asignaturismo (área de expresión, área de iniciación a la tecnología, etc), la creación de un espacio de actividades planificadas optativas (APO), y la introducción - por parte del docente- de métodos de trabajo activos. En este plan se estableció la coordinación de asignaturas dentro de las áreas tratando de evitar el excesivo fraccionamiento de los conocimientos (ANEP, 1994, p. 41)

En esta época, la Ley de emergencia para la enseñanza 15.739, estableció en su articulo 11 el cometido de ANEP que será responsable de "Organizar y realizar, a nivel terciario, en todo el territorio de la república la formación y perfeccionamiento del personal docente”.

En el marco jurídico de esta ley, se destacamos el Art. 606 que dispuso la creación del cargo de Directo Ejecutivo del Área de Formación y Perfeccionamiento Docente. Unos años mas tarde, en 1990 Acta 47 Res. nº 87 23/8 de 1990 definió las atribuciones de cargo de Director Ejecutivo del Área de Formación y Perfeccionamiento Docente crea los cargos de subdirectores del Área media y Técnica y del Área Magisterial. Este organismo es el responsable de la formación docente hasta la creación del Consejo de Formación en Educación en la Ley 18537 del 2008.

Asimismo, es relevante resaltar que en este período se le otorga potestad legal a las 
asambleas técnicas de docentes, (ATD), q en el capitulo VII estatuto del funcionario docente impone el establecimiento de las Asambleas de docentes de los Institutos, Liceos y Escuelas de su dependencia (de la ANEP) así como Asambleas nacionales de docentes de cada Consejo desconcentrado. Las mismas tendrán derecho de iniciativa y función consultiva en los problemas técnico-pedagógicos de la rama respectiva y en temas de educación general. Corresponderá su reglamentación al Consejo Directivo Central ${ }^{2}$.

Diferentes estudios encomendados por el CODICEN de la ANEP, hallaron debilidades en las políticas de formación en servicio de los docentes, como por ejemplo el oficio ANEP( $\mathrm{N}^{\mathrm{o}}$ 582 , p.19) en referencia al único Instituto de formación de profesores para educación media de la época, el IPA, señalaba que "de las funciones asignadas originariamente al IPA, ha cumplido en los últimos años sólo las de la formación inicial del profesores de Educación Media, habiendo dejado de cumplir funciones de perfeccionamiento, de especialización y de promoción de la investigación”. Un indicador elocuente es que la cuarta parte del profesorado de educación básica técnica no participó en ningún tipo curso de capacitación en servicio o actividad académica en el período 1990ç1994 (CEPAL, 1990,1991,1992).

Otro hallazgo de la Comisión Económica para América Latina y el Caribe (CEPAL) fue advertir que el panorama del profesorado de educación básica técnica era particularmente problemático: existía un bajo compromiso institucional derivado del multiempleo así como un nivel muy bajo de titulación pedagógica especifica del personal docente, (apenas $15 \%$ del profesorado)

En síntesis, al año 1995, el personal docente de educación básica uruguayo no sólo carece de formación pedagógica, y del estímulo para el uso de las herramientas didácticas necesarias para una docencia de calidad, sino que tampoco el Estado ha brindado las oportunidades de capacitación en servicio, actualización y formación permanente.

Ni los esfuerzos para mejorar la estructura edilicia y material de la enseñanza, ni las

\footnotetext{
${ }^{2}$ En el sistema público uruguayo, el ingreso al ejercicio de la docencia en ANEP se encuentra regulado actualmente en el Estatuto del funcionario docente, Ordenanza N..$^{\circ} 45$ (aprobado por Acta No 68 , Resolución N. ${ }^{\circ} 9$ del 20 de diciembre de 1993, y modificado por las Resoluciones del Consejo Directivo Central al 31 de julio de 2008)
} 
nuevas reformas políticas impulsadas como el nuevo Plan curricular del año 1986 orientadas a cambiar el funcionamiento institucional de los liceos lograron detener la profundización de la crisis. Una de las causas fundamentales analizadas por la normativa jurídica del próximo gobierno de la educación (1995-1999) es la que determinó la necesidad crear un modelo de formación de docentes de educación básica que promoviera un cambio significativo en las prácticas para revertir los altos niveles de inequidad del sistema uruguayo.

\section{El periodo 1996-2008: reformas integrales y nuevas bases jurídicas de las políticas docentes para educación básica}

Las políticas para educación básica posteriores a la caída de la dictadura, se caracterizaron por un fuerte impulso a reformas educativas integrales y por el impulso de una renovación profunda de la legislación educativa a partir de la aprobación en el año 2008 de la Ley de General de Educación.

A inicio de este periodo, entre los años 1990 y 1995, el gobierno, a través de los entes autónomos, promulgó diferentes iniciativas y programas para realizar una evaluación de planes curriculares y múltiples diagnósticos educativos de las modalidades de formación implementadas para educación básica en un contexto general de desajuste histórico entre el crecimiento de la matricula escolar (especialmente un crecimiento explosivo en educación secundaria) ${ }^{3}$ y las capacidades del sistema.

A partir del año 1996 se instrumentaron políticas que abarcaron transformacionales integrales en todo el sistema educativo (planes y programas de educación básica, estructura de la formación y capacitación, nuevos lineamientos para el trabajo docente, la gestión de los centros y la supervisión educativa). En esta época de restauración aún eran precarias las condiciones materiales de los trabajadores de la enseñanza (escasez de bibliotecas, falta de apoyo para la compra de libros, recursos para la docencia) y en particular, el bajo salario de la profesión docente en educación media.

Tal como ya fuera informado, la CEPAL, impulso varios diagnósticos educativos en este

\footnotetext{
3 Uruguay fue pionero en la región al decretar la obligatoriedad de la educación básica ,que incluía desde primaria hasta los primeros 3 años de educación media, ya en el año 1973.
} 
periodo $(1990,1991,1992)$. El referido a educación básica concluyó que “el sistema había llegado a una etapa de crisis profunda por el prolongado menoscabo de las remuneraciones y de las condiciones materiales de la educación. Este deterioro se profundizó en el período de la dictadura (1973-1984), que tanto por razones ideológicas como por competencia en el gasto público entre las asignaciones a la enseñanza o a las fuerzas armadas -los dos más voluminosos cuerpos de funcionarios del Estado- fue disminuyendo el porcentaje de gasto público y del PBI dedicado a la educación" (CEPAL, 1990,56).

La democratización del acceso al sistema educativo generó importantes niveles de inequidad, que en la primera mitad de la década de los 90 se manifestó en el crecimiento de la tasa de deserción en 1er año de educación media básica que alcanzó casi el 40 \% de los alumnos. A este periodo se lo denominó, la “democratización inconclusa” (Opertti, 2003).

En este contexto general de austeridad presupuestal, la prioridad política fue la evaluación del sistema educativo, el desarrollo de diagnósticos acerca de los resultados y la eficiencia del sistema, el establecimiento de un nuevo marco jurídico de regulación del trabajo docente, así como la promoción de la participación de los docentes en los órganos de conducción. Por oposición, las políticas de desarrollo profesional docente fueron casi inexistentes, al punto de que el país, hasta el año 1996, no dispondría de una agencia encargada de la capacitación en servicio de los docentes de educación básica.

El cambio de gobierno de la educación del año 1996 implicó el inicio de un conjunto significativo de iniciativas legales y normativas que apuntaron al desarrollo de la formación inicial y capacitación en servicio de los docentes de educación básica ${ }^{4}$.

El nuevo marco jurídico establecido en la Ley de Presupuesto Gastos e Inversiones $n^{\circ}$ 16.737 para el quinquenio 1996-2000, planteó cuatro objetivos estratégicos de política educativa: dignificar la formación y la función docente, el mejoramiento de la calidad de la educación, el avance en términos de equidad y la modernización de la gestión.

\footnotetext{
4 El problema de la escasez de profesores era muy grave, tal como señala un director de la época, entrevistado en el estudio de la CEPAL, “"Hay otro problema además que tiene que ver con los docentes del CBU y es la enorme afluencia de gente que no tiene formación docente y para los cuales el trabajo en secundaria es la changa del estudiante universitario mientras estudia” en CEPAL, ¿Aprenden los estudiantes? El Ciclo Básico de Educación Media, 1990, Pág.74.
} 
La creación de los centros regionales para la formación de profesores de educación media, en el interior del país, fue uno de los ejes principales de las reformas impulsadas entre los años 1997 y 2007. Estos centros responden a una estrategia de descentralización de la formación, se ubicaron en 6 polos regionales, donde se instaló la idea del polo regional y cultural a partir de un modelo de formación docente diferente al desarrollado por el IPA en Montevideo.

El nuevo modelo educativo para formar a los docentes de secundaria se basó en la alta carga horaria de los docentes formadores (40 horas semanales), becas para estudiantes de docencia, promoción del trabajo interdisciplinario, desarrollo de la investigación aplicada en educación, implementación de un nuevo currículo por áreas de conocimiento e incorporación de cursos en tecnología, idiomas y análisis de las prácticas educativas. Los centros regionales contribuyeron con la mejora en la profesionalización del ejercicio docente en educación media básica y superior. Los primeros datos de egreso respecto a esta innovación dieron cuenta de su incidencia en el crecimiento del $34 \%$ de los docentes titulados en el interior del país. Además, la elevada tasa de deserción en profesorado en 1er año que oscilara en el 60\% en el IPA, disminuyó hasta el 11\% con la regionalización ${ }^{5}$ (ANEP, 1994).

En paralelo a la reforma de la formación inicial, el plan curricular 96 para educación media básica introdujo cambios significativos en la organización de la gestión en los centros educativos de nivel medio. Se introduce la idea del trabajo docentes por áreas de conocimiento, con el apoyo de las ATD (ATD, 1996)). En este nuevo régimen de asignación de horas, fuertemente rechazado por los sindicatos docentes de secundaria ${ }^{6}$ los docentes eligen los cursos y el centro donde se desempeñarán en el año escolar en un régimen de contrato por 20 horas que incluía una novedosa modalidad de elección de horas según agrupamiento de asignaturas (ciencias sociales, por ejemplo, en lugar de optar únicamente por historia). Esta modalidad de elección estaba en consonancia con la formación interdisciplinaria que los profesores del interior del país recibían en los centros regionales (CERP) creados por esta nueva administración.

\footnotetext{
6 La enseñanza por áreas (ciencias sociales y ciencias de la naturaleza) implicaba abandonar el enfoque asignaturista en el curriculum de enseñanza media para pasar a un abordaje de tipo interdisciplinario. Fue una de las innovaciones más resistidas por los docentes e inspectores de educación media básica. La Federación Nacional de Profesores de Secundaria (FENAPES) Según la opinión de Spadoni, miembro ejecutivo de FENAPES en los 90 , este modelo de reforma consta de tres aspectos básicos: "una visión economicista de la educación, un proyecto de mercado para el cual hay que preparar al alumno y la intención de hacer rendir los recursos humanos al máximo" . Semanario Brecha, "Cuestionamiento en Rama", 15/9/1995, p.7.
} 
En este marco general de reforma integral, se creo el Centro de Capacitación n y Perfeccionamiento Docente (CECAP), con sede en Montevideo, responsable de la capacitación $\mathrm{n}$ en servicio de los maestros y profesores del sistema (ANEP-CODICEN, Proyecto de Ley Presupuestal 1996-2000, Tomo IV).

Uno de los méritos de la Reforma Educativa fue el desarrollo de políticas de capacitación a docentes de educación básica que por primera vez se realizaban de forma masiva. Los cursos tenían como propósito mostrar que las innovaciones y las iniciativas de mejora de la calidad de la enseñanza en todos los subsistemas de la ANEP estaban articuladas entre si (cambios en los programas de formación, mejora de calidad educativa en todos los niveles y nuevos planes curriculares, por ejemplo). La particularidad de esta política fue asumir la necesidad de destinar fondos para cubrir el alto costo de la formación masiva de docentes (por causa del traslado a la capital y generación de costos de residencia y viáticos de estadía para los docentes que participaban de los programas en el CECAP de Montevideo). Además, el formato de capacitación implicó la concentración de una alta carga horaria de formación en corto tiempo y la utilización por primera vez en el país, de la modalidad semi presencial de formación.

Asimismo, se impulsó una fuerte política de capacitación hacia maestros, directores escolares e inspectores de este nuevo formato escolar. Se crean las escuelas de tiempo completo (ECC), un nuevo modelo pedagógico y organizacional de las escuelas de primaria, (Res. 21, Acta 90, 1998) ${ }^{7}$.

En el año 2000 al igual que en Brasil, el país apostó a desarrollar una propuesta institucionalizada de uso de la tecnología para la formación en servicio y formación de docentes a distancia, con la instalación del profesorado semipresencial. En el el año 2000 se creó el Grupo de Trabajo Inicial de Educación a Distancia y Nuevas Tecnologías. (ANEP, CODICEN, Acta No 83 Res. 11. 14 de noviembre de 2000). A partir del 2003 esta oferta se desarrolla a nivel de todo el territorio nacional en los centros centros de formación de maestros descentralizados (IFD). En el año 2018 se conocen sus resultados en términos de eficiencia y logros alcanzados. (CFE, Acta N² Res. N 9, Exp. 2018-25-5-000 44Qvp).

\footnotetext{
7 Estas escuelas surgieron como una necesidad de amortiguar las experiencias negativas del entorno social y territorial de los niños en situaciones de pobreza. La normativa dispone que los docentes en estas escuelas trabajan en el marco de un proyecto pedagógico institucional, durante 40 horas semanales, de las cuales 2 horas y media están destinadas a reunión de intercambio y planificación.
} 
Dos reportes de investigación de reciente divulgación (Conde, Gonzalez, Villagran, 2018, Rodriguez Zidán y Grilli, 2019 ) dan cuenta de una baja tasa de titulación de esta modalidad curricular a distancia: $6,7 \%$ de titulados en una matricula total de 3.813 estudiantes matriculados en el año 2017. Los estudios detectaron graves problemas de eficiente interna: débil capacitación de los docentes formadores en aulas virtuales, sub-utilización de la interfaz de las plataformas educativas y altos costos de sistema por el traslado de los estudiantes semi presenciales de todo el país que deben concurrir obligatoriamente a los encuentros nacionales organizados en la ciudad de Montevideo.

En esta época, el gobierno del partido Frente Amplio desarrolló una fuerte política pública de tecnología educativa, equidad digital, acceso a Internet gratuito y gobierno electrónico, liderada por el Plan Ceibal (Conectividad Educativa de Informática Básica para el Aprendizaje en Línea).

Ceibal se instaló en el año 2007 y desde el primer momento se proyectó como innovación educativa, vista por referentes y organismos internacionales como un ejemplo a seguir por la distribución universal de dispositivos (laptops y tabletas), así como por implementar el uso de plataformas de aprendizaje, aplicaciones, recursos digitales (Biblioteca Ceibal) y evaluaciones en línea, entre otros múltiples recursos que se mantienen hasta la actualidad. Este centro continúa ocupando un lugar destacado en la formación en servicio de los maestros y profesores de educación básica en tecnologías digitales para la educación y en el uso de plataformas de enseñanza a través del Centro Ceibal de Formación, el componente de formación de Plan Ceibal capacitó 19.500 docentes entre 2014 y 2018.

\section{La Ley General de Educación 2008 y la nueva base legal de las políticas docentes}

La aprobación de la Ley General de Educación del año 2008 conjuntamente con las iniciativas del CFE en este periodo introduce cambios significativos tanto en la estructura del sistema educativo como en la formulación de políticas docentes. Desde el punto de vista formal, se avanzó en la instalación del CFE desde el año 2010. Entre sus cometidos tenía la obligación legal de "Concretar la instalación e implementación del Instituto Universitario de Educación” y "Asegurar la continuidad y superación de la calidad educativa de las acciones de formación de 
grado y posgrado de docentes y educadores y contribuir al desarrollo profesional permanente de los docentes y educadores en servicio".

Este organismo, entre los años 2015-2019 promovió cambios en la formación inicial y permanente de los profesores de educación básica, instaló nuevas propuestas de planes curriculares (maestros educación primaria plan 2010, maestros de educación primera infancia plan 2017 y profesorado técnico, plan 2017), y creó el IPES para capacitación de los docentes (en sustitución del CECAP, (Acta Extraordinaria $n^{\circ}$ 5, 2010, Res. $n^{\circ} 1$ del Consejo Directivo Central de la ANEP).

La capacitación de los docentes de educación básica, se ha desarrollado principalmente a nivel de programas nacionales. La formación a nivel de cada centro educativo son prácticas poco habituales en Uruguay, dado el fuerte centralismo de las políticas (salvo algunas excepciones como el proyecto PROMEJORA en educación media, en ocasiones los centros regionales de profesores o algunas escuelas de primaria referentes ${ }^{8}$.

El IPES es la institución principal para el desarrollo profesional de los docentes de educación básica. En general los cursos que ofrece, tanto para educadores de primera infancia o primaria como de secundaria, están centrados en la formación didáctica, organización y gestión de centros educativos y actualización de contenidos disciplinares. La mayoría de la oferta de seminarios, talleres y cursos ofrecidos en el IPES no establecen requisitos de evaluación para su aprobación.

Por otra parte, de la Ley General de Educación aprobada en el año 2008, destacamos, la formulación de una política de educación terciaria universitaria (Artículo 30), la creación del Instituto Universitario de Educación (IUDE) en el ámbito del Sistema Nacional de Educación Pública que desarrollará actividades de enseñanza, investigación y extensión que formará maestros, maestros técnicos, educadores sociales y profesores, y otorgará otras titulaciones que la educación nacional requiera. (Artículo 84) y del Instituto Terciario Superior (ITS) en el

\footnotetext{
${ }^{8}$ Como el caso del CEIP q en 2015 destinó fondos para el desarrollo de proyectos nivel de centro para le desarrollo de competencias en matemática y lengua, (Circular $n^{\circ} 41$ del Consejo de Educación Inicial y Primaria ) y algunos experiencias de formación en servicio que impulsaron los centros regionales de profesores..
} 
ámbito del Sistema Nacional de Educación Pública. (Artículo 87) que estará constituido por una red de instituciones ubicadas en diferentes lugares del país. Este nuevo instituto desarrollará actividades de educación terciaria, integrando enseñanza, investigación y extensión.

Respecto a las políticas docentes establecidas por las diferentes Leyes de Presupuesto analizadas y por el marco jurídico de la Ley de educación vigente, Uruguay avanzó en el diseño de planes y programas, en la formulación de políticas con la participación docente en los entes de gobierno. En cambio, no ha logrado mejorar sustantivamente problemas estructurales vinculados con la profesión docente.

Aun hoy, la exigencia de la obligatoriedad del título para el ejercicio docente en $7 \mathrm{mo}$, 8vo y 9noo (artículo 69, Ley General de Educación no 18.43), no se cumple, ya que se continúa contratando estudiantes de docencia o universitarios para ejercer la enseñanza en el nivel medio. En el mismo sentido, la creación del Instituto Universitario para la formación docente (artículo 84, Ley General de Educación $n^{\circ} 18.43$ ) no ha avanzado mas allá de su formulación legal.

Si bien la Ley General de Educación dispone la transformación de la formación docente en universitaria, la aprobación de nuevas normativas e instrumentos para crear efectivamente este nuevo instituto no se alcanzó por falta de consenso parlamentario y acuerdo entre los actores educativos 9 .

Por diferentes razones, que la investigación atribuye a factores de inercia institucional, apego a modelos tradicionales de gobierno, gestión y métodos de enseñanza, y ausencia de consensos políticos necesarios para legislar e instrumentar la estructura funcional y organizativa de una institución superior de docencia o universidad pedagógica (Mancebo, 2019, Coutinho, 2017), los avances legislativos de los últimos 12 años no son suficientes para lograr una transformación profunda de las políticas docentes en educación básica.

\footnotetext{
9 Para las Asambleas Técnico Docente del CFE el proyecto del IUDE no cubría sus aspiraciones de cogobierno y autonomía, por lo que resolvieron apoyar la creación de una Universidad de la Educación autónoma y cogobernada. Varios sectores del partido Frente amplio apoyaron esta iniciativa, que no prosperó en el parlamento nacional ya que requería mayorías especiales. Véase:

http://www.cfe.edu.uy/index.php/cfe-en-la-prensa/3348-fa-insistira-con-proyecto-de-ley-de-creacion-de-la-universidad-de-la-educacion
} 
A manera de síntesis, presentamos en la figura 1 los principales hitos de las políticas docentes de los últimos 35 años en Uruguay así como las líneas de política docente y el marco legal que las sustenta.

\begin{tabular}{|c|c|c|c|}
\hline \multicolumn{4}{|c|}{$\begin{array}{c}\text { Figura 1. Políticas docentes y base normativa del sistema educativo uruguayo (años } \\
\text { seleccionados) }\end{array}$} \\
\hline $\begin{array}{l}\text { Período } \\
\text { histórico }\end{array}$ & $\begin{array}{l}\text { Hitos de las } \\
\text { políticas } \\
\text { Docentes } \\
\end{array}$ & $\begin{array}{l}\text { Líneas de Política } \\
\text { Docente en EB }\end{array}$ & Base Normativa \\
\hline 1985-1994 & $\begin{array}{l}\text { Recuperación } \\
\text { democrática } \\
\text { Estatuto Docente. } \\
\text { Gobierno de la } \\
\text { educación }\end{array}$ & $\begin{array}{llll}\text { Plan Curricular } & 86 & \text { para } & \text { Ciclo } \\
\text { Básico }(7,8 \text { y 9no) } & & \\
\text { Plan Curricular } & 1992 & \text { para } \\
\text { Magisterio } & & \end{array}$ & $\begin{array}{l}\text { Ley de emergencia Nero } \mathrm{N}^{\circ} 15.739 \text {, } \\
\text { Ley } 16.115 \text { (procedimiento para } \\
\text { elegir a las autoridades de la ANEP) } \\
\text { Ley de Presupuesto Gastos e } \\
\text { Inversiones } \mathrm{N}^{\circ} 16.737\end{array}$ \\
\hline 1995-2007 & $\begin{array}{l}\text {-Reforma } \\
\text { educativa } \\
\text {-Gobierno de la } \\
\text { Educación }\end{array}$ & $\begin{array}{l}\text { Plan Curricular } 1996 \\
\text { CERP Modelo descentralizado de } \\
\text { formación de Profesores de } \\
\text { educación básica y media superior } \\
\text { Centro de Capacitación CECAP } \\
\text { Profesorado Semi presencial } \\
\text { Congreso Nacional Educación Julio } \\
\text { Castro (2005) }\end{array}$ & 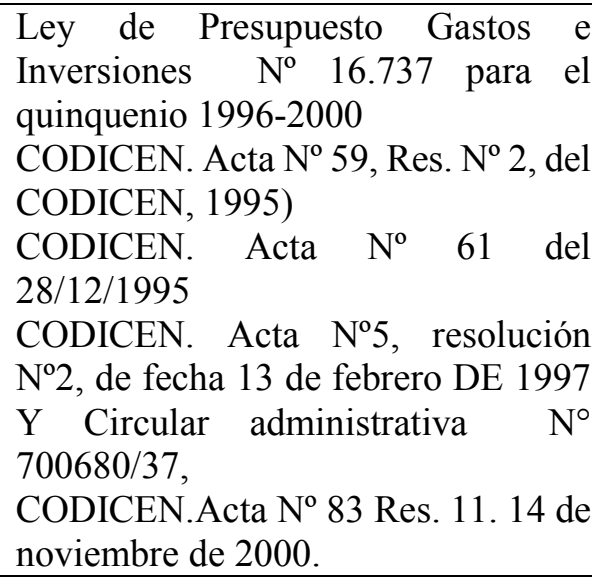 \\
\hline 2008- 2019 & $\begin{array}{l}\text {-Hacia un modelo } \\
\text { universitario de la } \\
\text { formación } \\
\text { docente (IUDE- } \\
\text { Universidad de la } \\
\text { Educación) } \\
\text {-Gobierno de la } \\
\text { Educación }\end{array}$ & 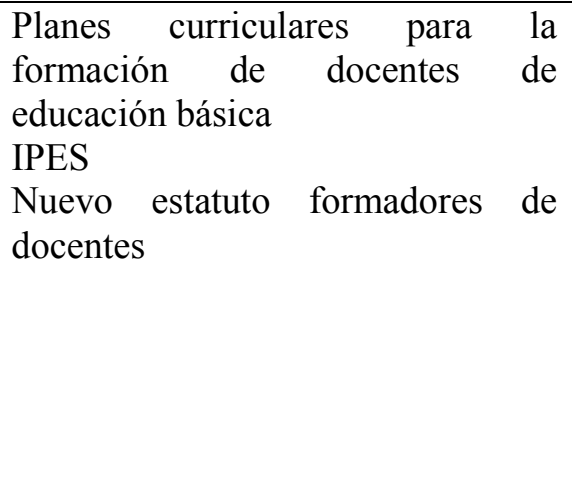 & $\begin{array}{l}\text { Ley General de Educación } 18.437 \\
\text { CFE. Acta } N^{\circ} 32 \text {. Res. } 29 \text { de } 23 \text { de } \\
\text { agosto de } 2017 \\
\text { CFE. Acta } N^{\circ} 44 \text {, Res. No } 36 \text { del } 5 \text { de } \\
\text { diciembre de } 2017 \\
\text { CFE. Acta } N^{\circ} 44 \text {, Res. } N^{\circ} 48 \text { de } 4 \text { de } \\
\text { diciembre de } 2018 \text {. } \\
\text { CFE. Acta } N^{\circ} 5 \text {, Res. No } 9 \text { del } 12 \text { de } \\
\text { abril de } 2018 \\
\text { CFE. Acta } N^{\circ} 31 \text {, Res. No } 41 \text { del } 3 \text { de } \\
\text { setiembre de } 2019 \text {. }\end{array}$ \\
\hline 2020 & $\begin{array}{l}\text { Hacia un carácter } \\
\text { universitario de la } \\
\text { FD } \\
\text { Gobierno de la } \\
\text { Educación }\end{array}$ & $\begin{array}{l}\text { Programa MEC de desarrollo } \\
\text { profesional } \\
\text { Convocatoria voluntaria para los } \\
\text { centros de profesorado } \\
\text { Cambios en la constitución de los } \\
\text { desconcentrados de la ANEP }\end{array}$ & $\begin{array}{l}\text { Ley de Urgente Consideración, Nero } \\
\text { 19.889, sección III, Educación }\end{array}$ \\
\hline
\end{tabular}


El 1 de marzo de 2020, asumieron las nuevas autoridades en educación para el período 2020-2025. Tres ideas fuerza del nuevo marco jurídico apuntan al desarrollo de reformas institucionales y transformaciones de la formación de los docentes de educación básica: el CODICEN de la ANEP deberá promover acciones tendientes a la creación de una formación universitaria de la educación (articulo 170, LUC), se innova al incluir un procedimiento voluntario de reconocimiento ante el MEC del nivel universitario de carreras de formación docente impartidas por entidades públicas no universitarias (articulo 198, LUC) y se propone la creación de un Programa Nacional de Fortalecimiento de la Formación en Educación también en la órbita del MEC (articulo 85, LUC).

Todo indica que los nuevos desafíos para las políticas docentes en educación básica en el periodo 2020-2025 se centrarán en avanzar en tres direcciones. La transformación del carácter universitario de la formación de docentes, atender las debilidades del modelo de formación inicial docente, (creando nuevos instrumentos de política para la formación compensatoria del $40 \%$ de los docentes de educación básica que no tienen titulo de profesor) y llevar adelante de manera efectiva un programa de desarrollo profesional para los docentes de primaria y secundaria básica que estimule la formación académica como instrumento de ascenso en la carrera docente.

\section{Para seguir reflexionando ...}

La discusión sobre políticas públicas para la formación de docentes sigue siendo un tema central tanto en Brasil como en Uruguay. Con base en la documentación legislativa comparada, surge que el énfasis y los desafíos en cada sistema educativo tienen aspectos comunes pero también presentan prioridades diferentes.

En Brasil, incluso con avances significativos, los discursos todavía están disfrazados y no son visibles: al repetir la afirmación de la importancia de la educación y la preparación de los docentes para la Educación Básica, aumenta el número de proyectos aligerados y sin una base coherente con la realidad brasileña, tanto para estudiantes como para docentes.

El análisis de los marcos legales para la formación de docentes en Brasil, además de señalar la alineación con las directrices de las organizaciones internacionales, muestra formas 
de interferencia directa en las prácticas educativas desarrolladas por los docentes a través de la educación continua. Los documentos oficiales se apropian de los mismos conceptos y lineamientos metodológicos presentes en las propuestas de las organizaciones multilaterales para la formación de docentes. Centrados en la capacitación por habilidades y en la práctica, fomentan la reducción del trabajo educativo a una práctica acrítica en lugar de capacitación teórica y académica. No es posible desarrollar Directrices sin un conjunto completo de condiciones de trabajo y capacitación garantizadas. La formación de docentes en la universidad debe ser el resultado de un proyecto específico que comparten todos los docentes de todos los grados. También significa involucrar a toda la sociedad y sus educadores e investigadores en este proceso formativo, analizando la realidad y recreando alternativas para una acción políticopedagógica más consistente y coherente.

Por otra parte, Uruguay, colocó en el centro de las políticas el diseño institucional del gobierno de la enseñanza, la renovación de planes y programas, emprendiendo acciones y orientando el presupuesto educativo para el aseguramiento del acceso a la educación con inclusión social y el desarrollo de innovaciones en tecnologías educativas a partir del Plan Ceibal. No obstante, no ha logrado superar el problema de la contratación temporal de docentes de educación básica, reducir el porcentaje de docentes sin titulación, instalar una política integral de formación permanente y avanzar firmemente hacia una formación docente universitaria.

En ambos casos, tanto los avances parciales, como los desafíos pendientes, plantean retos para continuar con la investigación educativa en temas de alto interés nacional como el aseguramiento de una educación básica de calidad, integral, y sin exclusiones.

\section{BIBLIOGRAFIA}

ANEP (2019). Educación en el territorio Uruguay, (agosto 2019), recuperado de http://ccdd.anep.edu.uy/sites/default/files/2019-08/Informe $\% 20 \mathrm{pa} \% \mathrm{C} 3 \%$ ADs.pdf

ANEP CFE (2020). Consejo de Educación en Formación. MemorIa 2015-2019.ANEP

ANEP (2019). Censo Nacional Docente 2018. Informe de resultados, recuperado de https://censodocente2018.anep.edu.uy/censo/ ANEP. (2015). 
ANEP.CFE. (2019). Plan de Formación para la carrera de Profesoradoen todas susespecialidades. Expediente $\mathrm{N}^{\circ}$ : 2019-255-007977, setiembre2019.

ANEP, "La renovación educativa. Educación Media", ANEP.

ANEP- CODICEN (1995). Proyecto de Ley Presupuestal 1996-2000, Tomo IV.

ANEP (2000), “Una visión integral del proceso de reforma educativa en Uruguay 1995-1999”. ANEP

ATD Nacional IX (1996), “Una Apuesta a la participación I”, Solís marzo 1996, pág. 46 y 50.

ANEP, CFE, (2018), informe 2017 Estudio sobre la modalidad de formación semipresencial del Consejo de Formación en Educación,

ANEP. (2015d). Proyecto de Presupuesto Período 2015-2019. Tomo I - Exposición de Motivos. Recuperado de https://www.anep.edu.uy/sites/default/files/images/Archivos/presupuestosrendicionbalances/Proyecto de Presupuesto Período 2015-2019 - Tomo I - Exposición de Motivos.pdf.

ARROYO, M. (2015). Os Movimentos Sociais e a construção de outros currículos. Educar em Revista. Editora UFPR: Curitiba, Brasil, n. 55, p. 47-68, jan./mar.

BRASIL (1996), Lei de Diretrizes e Bases. Lei n 9394/96, 20 de dezembro de 1996. Disponível em <http:/www.planalto.gov.br/ccivil_03/Leis/L9394.htm> acesso em: 20/03/2020.

BRASIL (2014). Lei no 13.005, de 25 de junho de 2014. Aprova o Plano Nacional de Educação (PNE) e dá outras providências. Diário Oficial da União [da] República Federativa do Brasil, Brasília, 2014. Disponível em <http://www.planalto.gov.br/ccivil_03/_Ato20112014/2014/Lei/L13005.htm> acesso em: 20/03/2020.

BRASIL (2015). Conselho Nacional de Educação. Conselho Pleno. Resolução n²/2015. Define as Diretrizes Curriculares para a formação inicial em nível superior (cursos de licenciatura, cursos de formação pedagógica para graduados e cursos de segunda licenciatura) e para a formação continuada. Brasília, DF: CNE, 2015. Disponível em $<$ http://portal.mec.gov.br/docman/agosto-2017-pdf/70431-res-cne-cp-002-03072015-pdf/file> acesso em: 20/03/2020.

BRZEZINSKI. I. (2017). Segunda licenciatura em dança do PARFOR: da cidadania consentida à cidadania negada. Revista de Educação. Puc-Campinas. V.22; n.2; pp.255-268.

CEPAL (1990). “Enseñanza Primaria y Ciclo Básico en Uruguay. Montevideo CEPAL. CEPA”. 1991 "Qué aprenden y quiénes aprenden en las Escuelas del Uruguay. Los contextos institucionales de éxitos y fracasos”. Montevideo, CEPAL 1991.

CEPAL (1991) ¿Aprenden los estudiantes? El ciclo Básico de Educación Media. Montevideo. 
CEPAL (1992). Los Bachilleres Uruguayos: quiénes son, que aprendieron y que opinan. Montevideo.

CIDE. (1965) COMISIÓN DE INVERSIONES Y DESARROLLO ECONÓMICO, Informe sobre el estado de la educación en Uruguay, tomo primero, Montevideo.

CASTAING, M. (2019) La investigación en Formación en Educación. Agencia Nacional de Investigación e Innovación (ANII). Consejo de Formación en Educación (CFE)

Conde, A, Gonzalez,M, Villagrán,A. (2018) Estudio sobre la modalidad de formación semipresencial del consejo de formación en educación (cfe). Informe, 2017

COITINHO SÁNCHEZ, A. V (2017). Las políticas públicas de profesionalización de 'formadores' en el Uruguay en los períodos de gobierno de Mujica y Vázquez (2005-2015): resistencias y bloqueos para avanzar en la profesionalización de los formadores. Revista.Transmutare, Curitiba, Vol. 2, N² 2017, p. 150-184.

GATTI, B.A. et al. (2011). Políticas docentes no Brasil: um estado da arte. Brasília, DF: UNESCO.

MANCEBO, MA. E. (2007). La educación uruguaya en una encrucijada: entre la inercia, la restauración y la innovación. En Bentancur, Nicolás (Org.). Las políticas educativas en Uruguay. Perspectivas académicas y compromisos políticos. Montevideo: Ministerio de Educación y Cultura, p. 8-42. ISBN: 9789-97436.

MEC (2013). Desarrollo Profesional Docente y Mejora de la Educación. Informe País. Ministerio de Educación y Cultura. Montevideo.

OLIVEIRA, H. L. G.; LEIRO, A. C,.R. (2019) Políticas de formação de professores no Brasil: referenciais legais em foco. Revista Pro-Posições. Epub Apr vol.30, e20170086.

OPERTTI, R. VILlagran, A. (2003) Revista de Ciencias Sociales. Departamento de Sociología. Año XVI / Nº $21 \approx$ AGOSTO 2003

RODRIGUEZ ZIDAN, E, GRILLI, J. (2019). El b-learning en la educación terciaria Estudio de las percepciones de docentes y estudiantes sobre la apropiación y el uso de las plataformas digitales en la formación inicial de profesores. ANEP.CFE. recuperado de http://repositorio.cfe.edu.uy/handle/123456789/486

\section{Legislación consultada}

Ley de Presupuesto, Gastos e Inversiones Nero 15.739 para el quinquenio 1986-1990

Ley de Presupuesto Gastos e Inversiones no 16.737 para el quinquenio 1996-2000

Ley General de Educación Nero 18.437 (2008) 
Ley de Urgente Consideración Nero 19.889 para el quinquenio 2020-2025

Lei n ${ }^{\circ}$ 9.394, de 20 de dezembro de 1996 (1996). Estabelece as diretrizes e bases da educação nacional. Recuperado em 20 de abril de 2020, de http://www.planalto.gov.br/ccivil_03/Leis/L9394.htm

Lei $n^{\circ} 13.005$, de 25 de junho de 2014 (2014). Aprova o Plano Nacional de Educação - PNE e dá outras providências. Recuperado em 20 de abril de 2020, de http://www.planalto.gov.br/ccivil_03/_ato2011-2014/2014/lei/113005.htm.

Resolução CNE/CEB n ${ }^{\text {2 }}$, de 19 de abril de 1999 (1999). Institui Diretrizes Curriculares Nacionais para a Formação de Docentes da Educação Infantil e dos anos iniciais do Ensino Fundamental, em Nível Médio, na modalidade Normal. Brasília: Conselho Nacional de Educação. Recuperado em 20 de abril de 2020, de http://portal.mec.gov.br/cne/arquivos/pdf/rceb02 99.pdf

Resolução CNE/CP n ${ }^{\circ}$ 02, de $1^{\circ}$ de julho de 2015 (2015). Define as Diretrizes Curriculares Nacionais para a formação inicial em nível superior (cursos de licenciatura, cursos de formação pedagógica para graduados e cursos de segunda licenciatura) e para a formação continuada. Brasília: Conselho Nacional de Educação. Recuperado em 20 de abril de 2020, de http://portal.mec.gov.br/index.php?option=com_docman\&view= download\&alias=17719-rescne-cp-002-03072015\&category_slug=julho-2015-pdf\&Itemid=30192

RESOLUÇÃO CNE/CP No 2, DE 20 DE DEZEMBRO DE 2019 (*) (1) Define as Diretrizes Curriculares Nacionais para a Formação Inicial de Professores para a Educação Básica e institui a Base Nacional Comum para a Formação Inicial de Professores da Educação Básica (BNC-Formação). Recuperado em 24 de abril de 2020. http://portal.mec.gov.br/docman/dezembro-2019-pdf/135951-rcp002-19/file 